УДК 538.945

\title{
Effect of the Spin-orbit Coupling and Impurity Scattering on the Spin Resonance Peak in Three-orbital Model for Fe-based Superconductors
}

\author{
Maxim M. Korshunov* \\ Yuliya N. Togushova ${ }^{\dagger}$ \\ Siberian Federal University
} Svobodny, 79, Krasnoyarsk, 660041

Russia

Received 15.10.2017, received in revised form 18.11.2017, accepted 24.12.2017

Here we study a combined effect of the spin-orbit coupling and scattering on the nonmagnetic disorder on the formation of the spin resonance peak in iron-based superconductors. Spin susceptibility is calculated within the random phase approximation. The spin resonance peak becomes broader with the increase of disorder and its frequency also shifts. At the same time, the spin response in the $s_{ \pm}$state is different from that of the $s_{++}$state.

Keywords: Fe-based superconductors, spin-orbit coupling, impurity scattering, spin resonance peak. DOI: $10.17516 / 1997-1397-2018-11-1-108-116$.

One of the central problem in the field of Fe-based superconductors (FeBS) is the microscopic nature of the superconducting pairing. FeBS can be divided into two classes, pnictides and chalcogenides, see Refs. [1-4]. Basic element is always the square lattice of Fe. Iron is surrounded by As or $\mathrm{P}$ situated in the tetrahedral positions within the first class and by Se, Te, or $\mathrm{S}$ within the second class. Pnictides are represented by 1111 systems (which include LaFeAsO, $\mathrm{LaFePO}, \mathrm{Sr}_{2} \mathrm{VO}_{3} \mathrm{FeAs}$, etc.) and 111 systems (LiFeAs, LiFeP, etc.) with the single Fe layer per unit cell, and 122 systems containing two FeAs layers per unit cell ( $\mathrm{BaFe}_{2} \mathrm{As}_{2}, \mathrm{KFe}_{2} \mathrm{As}_{2}$, etc.). Chalcogenides can be of 11 type $\left(\mathrm{Fe}_{1-\delta} \mathrm{Se}, \mathrm{Fe}_{1+y} \mathrm{Te}_{1-x} \mathrm{Se}_{x}\right.$, and FeSe monolayers) and of 122 type $\left(\mathrm{KFe}_{2} \mathrm{Se}_{2}\right)$. Since conductivity is provided by the FeAs layer, the discussion of physics in terms of quasi two-dimensional system in most cases gives reasonable results [4].

Fe $d$-orbitals form the Fermi surface that consists of two hole sheets around the $\Gamma=(0,0)$ point and two electron sheets around the $(\pi, 0)$ and $(0, \pi)$ points of the 1-Fe Brillouin zone (BZ) corresponding to one iron per unit cell. Nesting between these two groups of sheets is the driving force for the spin-density wave (SDW) long-range stripe antiferromagnetic order in the undoped and slightly doped FeBS. SDW state is destroyed upon doping but the residual scattering with the wave vector $\mathbf{Q}$ connecting hole and electron pockets leads to the enhanced antiferromagnetic fluctuations. $\mathbf{Q}$ is equal to $(\pi, 0)$ or $(0, \pi)$ in the $1-\mathrm{Fe} \mathrm{BZ}$.

Different mechanisms of Cooper pairing result in different superconducting gap symmetry and structure [4]. The RPA-SF (random-phase approximation spin fluctuation) approach gives the extended $s$-wave gap that changes sign between hole and electron Fermi surface sheets, $s_{ \pm}$ state, as the main instability for the wide range of dopings [4-9]. On the other hand, orbital

\footnotetext{
*mkor@iph.krasn.ru

$\dagger$ ytogushova@sfu-kras.ru

(c) Siberian Federal University. All rights reserved
} 
fluctuations promote the order parameter to have the sign-preserving $s_{++}$symmetry [10]. Thus, probing the gap structure can help in elucidating the underlying mechanism. In this respect, inelastic neutron scattering is a powerful tool since the measured dynamical spin susceptibility $\chi(\mathbf{q}, \omega)$ in the superconducting state carries information about the gap structure.

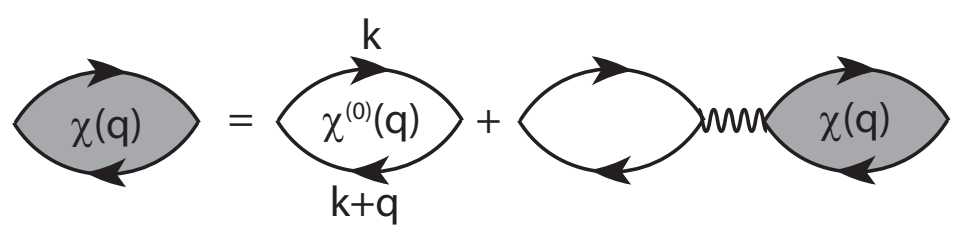

Fig. 1. RPA equation for the susceptibility

For the local interactions (Hubbard and Hund's exchange), $\chi$ can be obtained in the random phase approximation from the bare electron-hole bubble $\chi_{0}(\mathbf{q}, \omega)$ by summing up a series of ladder diagrams shown as a diagrammatic equation in Fig. 1. The solution to the equation is

$$
\chi(\mathbf{q}, \omega)=\left[I-U_{s} \chi_{0}(\mathbf{q}, \omega)\right]^{-1} \chi_{0}(\mathbf{q}, \omega),
$$

where $U_{s}$ and $I$ are interaction and unit matrices in orbital or band space, and all other quantities are matrices as well.

Scattering between nearly nested hole and electron Fermi surfaces in FeBS produce a peak in the normal state magnetic susceptibility at or near $\mathbf{q}=\mathbf{Q}$. For the uniform $s$-wave gap, $\operatorname{sign} \Delta_{\mathbf{k}}=\operatorname{sign} \Delta_{\mathbf{k}+\mathbf{Q}}$ and there is no resonance peak. For the $s_{ \pm}$order parameter as well as for an extended non-uniform $s$-wave symmetry, $\mathbf{Q}$ connects Fermi sheets with the different signs of gaps. This fulfills the resonance condition for the interband susceptibility, and the spin resonance peak is formed at a frequency $\omega_{s}$ below $\Omega_{c}=\min \left(\left|\Delta_{\mathbf{k}}\right|+\left|\Delta_{\mathbf{k}+\mathbf{q}}\right|\right)$. The existence of the spin resonance in FeBS was predicted theoretically $[11,12]$ and subsequently discovered experimentally with many reports of well-defined spin resonances in 1111, 122, and 11 systems [4]

There are two important ingredients that should be taken into account. One of them is the spin-orbit coupling. By introducing the spin-orbit (SO) interaction [13], it is possible to explain the observed anisotropy of the spin resonance peak in Ni-doped Ba-122 [14]. In particular, $\chi_{+-}$ and $2 \chi_{z z}$ components of the spin susceptibility are different thus breaking the spin-rotational invariance $\left\langle S_{+} S_{-}\right\rangle=2\left\langle S_{z} S_{z}\right\rangle$. Also, there are always some amount of disorder even in the best crystals. So the second important ingredient is the disorder. As the starting point, here we use the three-orbital model [15]. The combined effect of disorder and spin-orbit interaction on the spin susceptibility is studied via the static Born approximation for the quasiparticle self-energy due to the impurity scattering. Even such a simple approximation gives interesting qualitative results. Namely, the spin resonance becomes broader with the increase of disorder and its energy $\omega_{s}$ shifts to higher frequencies. Another result is that for the same amount of disorder the spin response in the $s_{ \pm}$state is still distinct from that of the $s_{++}$state, which is almost not affected by impurity scattering.

\section{Model and approach}

Here we use a simple three-orbital model for FeBS [15]. This model originates from the three $t_{2 g} d$-orbitals. The $x z$ and $y z$ components are hybridized and form two electron-like Fermi 
surface pockets around $(\pi, 0)$ and $(0, \pi)$ points, and one hole-like pocket around $\Gamma=(0,0)$ point. The $x y$ orbital is considered to be decoupled from them and form an outer hole pocket around $\Gamma$ point. Latter differs from some popular orbital models for FeBS $[4,6]$. However, according to angle-resolved photoemission (ARPES) data $[16,17]$ and density functional theory (DFT) calculations $[18,19], x y$ orbital contribution to the Fermi surface near $\Gamma$ point is sizable. This situation is simulated by introducing the $x y$ hole pocket near $\Gamma$ point in the three-orbital model.

The Hamiltonian is given by $H=H_{0}+H_{S O}$, where

$$
H_{0}=\sum_{\mathbf{k}, \sigma, l, m} \varepsilon_{\mathbf{k}}^{l m} c_{\mathbf{k} l \sigma}^{\dagger} c_{\mathbf{k} m \sigma}
$$

is one-electron part with $c_{\mathbf{k} m \sigma}$ being the annihilation operator of a particle with momentum $\mathbf{k}$, spin $\sigma$ and orbital index $m$.

The matrix of the one-electron energies has the following form:

$$
\hat{\varepsilon}_{\mathbf{k}}=\left(\begin{array}{ccc}
\varepsilon_{1 \mathbf{k}} & 0 & 0 \\
0 & \varepsilon_{2 \mathbf{k}} & \varepsilon_{4 \mathbf{k}} \\
0 & \varepsilon_{4 \mathbf{k}} & \varepsilon_{3 \mathbf{k}}
\end{array}\right),
$$

where

$$
\begin{aligned}
\varepsilon_{1 \mathbf{k}} & =\epsilon_{x y}-\mu+2 t_{x y}\left(\cos k_{x}+\cos k_{y}\right)+4 t_{x y}^{\prime} \cos k_{x} \cos k_{y}, \\
\varepsilon_{2 \mathbf{k}} & =\epsilon_{y z}-\mu+2 t_{x} \cos k_{x}+2 t_{y} \cos k_{y}+4 t^{\prime} \cos k_{x} \cos k_{y}+ \\
& +2 t^{\prime \prime}\left(\cos 2 k_{x}+\cos 2 k_{y}\right), \\
\varepsilon_{3 \mathbf{k}} & =\epsilon_{x z}-\mu+2 t_{y} \cos k_{x}+2 t_{x} \cos k_{y}+4 t^{\prime} \cos k_{x} \cos k_{y}+ \\
& +2 t^{\prime \prime}\left(\cos 2 k_{x}+\cos 2 k_{y}\right), \\
\varepsilon_{4 \mathbf{k}} & =4 t_{x z y z} \sin k_{x} / 2 \sin k_{y} / 2 .
\end{aligned}
$$

To reproduce the topology of the Fermi surface in FeBS, we choose the following parameters $($ in $\mathrm{eV}): \epsilon_{x y}=-0.70, \epsilon_{y z}=-0.34, \epsilon_{x z}=-0.34, t_{x y}=0.18, t_{x y}^{\prime}=0.06, t_{x}=0.26, t_{y}=-0.22$, $t^{\prime}=0.2, t^{\prime \prime}=-0.07, t_{x z y z}=0.38$.

It is possible to diagonalize the matrix (3) using a unitary transformation with the matrix

$$
\hat{U}=\left(\begin{array}{ccc}
1 & 0 & 0 \\
0 & u_{\mathbf{k}} & v_{\mathbf{k}} \\
0 & -v_{\mathbf{k}} & u_{\mathbf{k}}
\end{array}\right),
$$

where $u_{\mathbf{k}}^{2}+v_{\mathbf{k}}^{2}=1, u_{\mathbf{k}}^{2}=\frac{1}{2}\left(1+\frac{\left|g_{\mathbf{k}}\right|}{D_{\mathbf{k}}}\right)$ and $v_{\mathbf{k}}^{2}=\frac{1}{2}\left(1-\frac{\left|g_{\mathbf{k}}\right|}{D_{\mathbf{k}}}\right), g_{\mathbf{k}}=\left(\varepsilon_{2 \mathbf{k}}-\varepsilon_{3 \mathbf{k}}\right)^{2}, D_{\mathbf{k}}=$ $g_{\mathbf{k}}+4 \varepsilon_{4 \mathbf{k}}^{2}$. Applying the transformation, we obtain the energy dispersion, $E_{1 \mathbf{k}}=\varepsilon_{1 \mathbf{k}}$ and $E_{2,3 \mathbf{k}}=$ $\frac{1}{2}\left(\varepsilon_{2 \mathbf{k}}+\varepsilon_{3 \mathbf{k}} \pm \sqrt{D}\right)$

Number of electrons $n$ on a filled $d$-orbital is 6 and for the three-orbital model we assume that two orbitals are completely filled, so for doping concentration $x$ we have $n=4-x$. In Fig. 2, the band structure and the Fermi surface are shown for the undoped material, $x=0$. Fermi surface is similar to those obtained in DFT calculations and five-orbital models [6,7].

In the absence of the SO coupling, we can calculate the physical susceptibility for Matsubara frequency $\omega_{m}$ as $\chi_{0}\left(\mathbf{q}, \omega_{m}\right)=\chi_{0}^{11}\left(\mathbf{q}, \omega_{m}\right)+\chi_{0}^{22}\left(\mathbf{q}, \omega_{m}\right)+\chi_{0}^{23}\left(\mathbf{q}, \omega_{m}\right)+\chi_{0}^{32}\left(\mathbf{q}, \omega_{m}\right)+$ $\chi_{0}^{33}\left(\mathbf{q}, \omega_{m}\right)$, where intra- and interband susceptibilities are $\chi_{0}^{11}\left(\mathbf{q}, \omega_{m}\right)=\frac{1}{2} \sum_{\mathbf{k}} \xi_{1 \mathbf{k}+\mathbf{q}}^{1 \mathbf{k}}\left(\omega_{m}\right)$, 

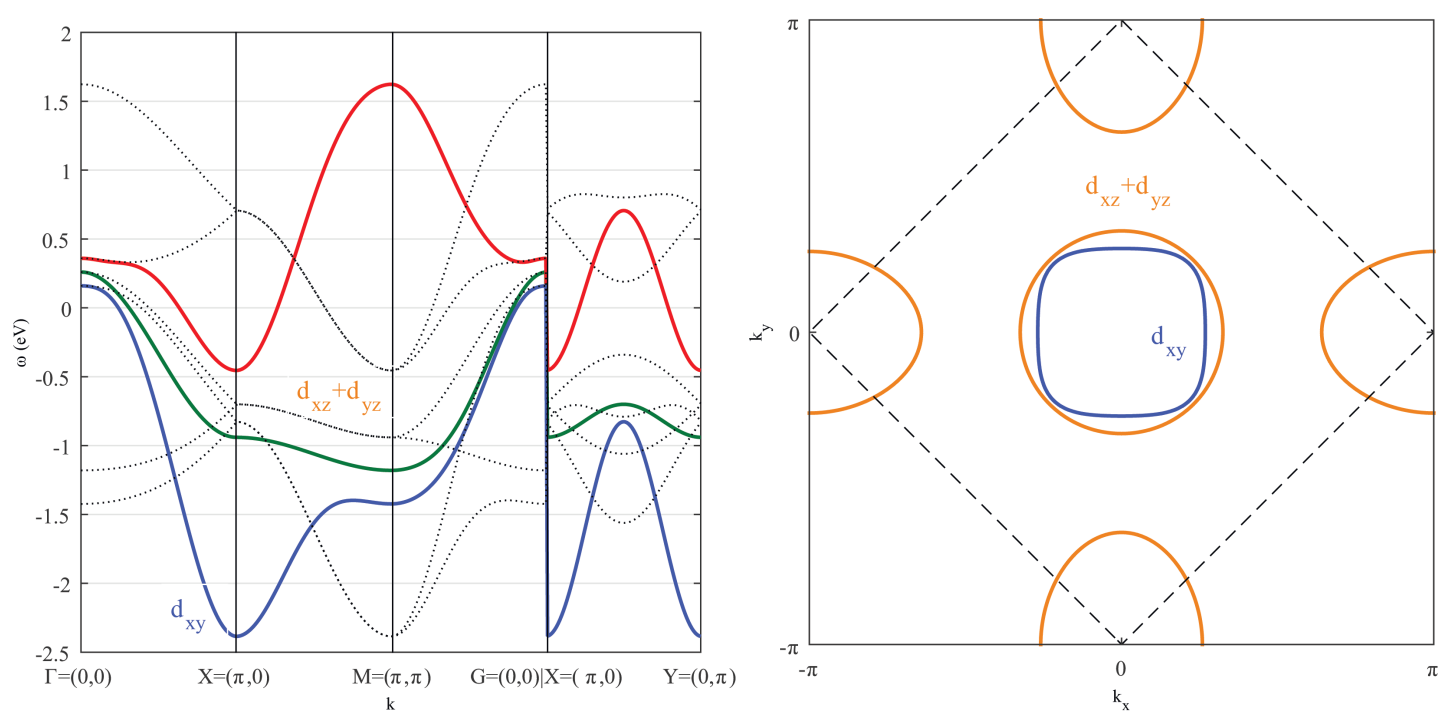

Fig. 2. Band dispersion (left, solid curves) and Fermi surface (right) for the three-orbital model in the 1-Fe BZ. Inner Fermi surface pocket around the $\Gamma$ point has a $d_{x y}$ orbital character, while other bands have a mixed $d_{x z}+d_{y z}$ character. Black dotted curves show bands dispersions in the 2-Fe BZ. Boundaries of the 2-Fe BZ are also shown as a rotated square in the FS plot

$\chi_{0}^{22}\left(\mathbf{q}, \omega_{m}\right)=\frac{1}{4} \sum_{\mathbf{k}}\left(1+\gamma_{\mathbf{k q}}\right) \xi_{2 \mathbf{k}+\mathbf{q}}^{2 \mathbf{k}}\left(\omega_{m}\right), \chi_{0}^{23}\left(\mathbf{q}, \omega_{m}\right)=\frac{1}{4} \sum_{\mathbf{k}}\left(1-\gamma_{\mathbf{k q}}\right) \xi_{3 \mathbf{k}+\mathbf{q}}^{2 \mathbf{k}}\left(\omega_{m}\right), \chi_{0}^{32}\left(\mathbf{q}, \omega_{m}\right)=$ $\frac{1}{4} \sum_{\mathbf{k}}\left(1-\gamma_{\mathbf{k q}}\right) \xi_{2 \mathbf{k}+\mathbf{q}}^{3 \mathbf{k}}\left(\omega_{m}\right), \quad \chi_{0}^{33}\left(\mathbf{q}, \omega_{m}\right)=\frac{1}{4} \sum_{\mathbf{k}}\left(1+\gamma_{\mathbf{k q}}\right) \xi_{3 \mathbf{k}+\mathbf{q}}^{3 \mathbf{k}}\left(\omega_{m}\right) . \quad$ Here $\xi_{\beta \mathbf{k}+\mathbf{q}}^{\alpha \mathbf{k}}\left(\omega_{m}\right)=$ $\frac{f\left(E_{\beta \mathbf{k}+\mathbf{q}}\right)-f\left(E_{\alpha \mathbf{k}}\right)}{\omega_{m}+E_{\alpha \mathbf{k}}-E_{\beta \mathbf{k}+\mathbf{q}}}, \quad \gamma_{\mathbf{k q}}=\frac{g_{\mathbf{k}} g_{\mathbf{k}+\mathbf{q}}+4 \varepsilon_{4 \mathbf{k}} \varepsilon_{4 \mathbf{k}+\mathbf{q}}}{D_{\mathbf{k}} D_{\mathbf{k}+\mathbf{q}}}$.

The three-orbital model for pnictides is similar to the one for $\mathrm{Sr}_{2} \mathrm{RuO}_{4}$. In particular, the $x y$ band does not hybridize with the $x z$ and $y z$ bands. Keeping in mind this similarity, we consider only the $L_{z}$-component of the SO interaction [20]. Due to the structure of the $L_{z}$-component, the interaction affects $x z$ and $y z$ bands only. Following Ref. [21], we write the SO coupling term, $H_{S O}=\lambda \sum_{f} \vec{L}_{f} \cdot \vec{S}_{f}$, in the second-quantized form as

$$
H_{S O}=\mathrm{i} \frac{\lambda}{2} \sum_{l, m, n} \epsilon_{l m n} \sum_{\mathbf{k}, \sigma, \sigma^{\prime}} c_{\mathbf{k} l \sigma}^{\dagger} c_{\mathbf{k} m \sigma^{\prime}} \hat{\sigma}_{\sigma \sigma^{\prime}}^{n}
$$

where $\epsilon_{l m n}$ is the completely antisymmetric tensor, indices $\{l, m, n\}$ take values $\{x, y, z\} \leftrightarrow$ $\left\{d_{y z}, d_{z x}, d_{x y}\right\} \leftrightarrow\{2,3,1\}$, and $\hat{\sigma}_{\sigma \sigma^{\prime}}^{n}$ are the Pauli spin matrices.

The matrix of the Hamiltonian $H$ then becomes

$$
\hat{\varepsilon}_{\mathbf{k} \sigma}=\left(\begin{array}{ccc}
\varepsilon_{1 \mathbf{k}} & 0 & 0 \\
0 & \varepsilon_{2 \mathbf{k}} & \varepsilon_{4 \mathbf{k}}+\mathrm{i} \frac{\lambda}{2} \operatorname{sign} \sigma \\
0 & \varepsilon_{4 \mathbf{k}}-\mathrm{i} \frac{\lambda}{2} \operatorname{sign} \sigma & \varepsilon_{3 \mathbf{k}}
\end{array}\right) .
$$


Corresponding eigenvalues are

$$
\begin{aligned}
E_{1 \mathbf{k}} & =\varepsilon_{1 \mathbf{k}}, \\
E_{2,3 \mathbf{k}} & =\frac{\varepsilon_{2 \mathbf{k}}+\varepsilon_{3 \mathbf{k}}}{2} \pm \sqrt{\left(\frac{\varepsilon_{2 \mathbf{k}}-\varepsilon_{3 \mathbf{k}}}{2}\right)^{2}+\varepsilon_{4 \mathbf{k}}^{2}+\frac{\lambda^{2}}{4}} .
\end{aligned}
$$

As in the case of $\mathrm{Sr}_{2} \mathrm{RuO}_{4}$, eigenvalues of $\hat{\varepsilon}_{\mathbf{k} \sigma}$ do not depend on spin $\sigma$, therefore, spin-up and spin-down states are still degenerate in spite of the SO interaction.

Components of the bare spin susceptibility have the following form (see [9] for details),

$$
\begin{aligned}
\chi_{(0) z z}^{l l^{\prime}, m m^{\prime}}\left(\mathbf{q}, \mathrm{i} \omega_{m}\right)= & -\frac{T}{4} \sum_{\omega_{n}, \mathbf{p}, \sigma}\left[G_{m l \sigma}\left(\mathbf{p}, \mathrm{i} \omega_{n}\right) G_{l^{\prime} m^{\prime} \sigma}\left(\mathbf{p}+\mathbf{q}, \mathrm{i} \omega_{m}+\mathrm{i} \omega_{n}\right)+\right. \\
& \left.+F_{l m^{\prime} \sigma}^{\dagger}\left(\mathbf{p},-\mathrm{i} \omega_{n}\right) F_{l^{\prime} m \sigma}\left(\mathbf{p}+\mathbf{q}, \mathrm{i} \omega_{m}+\mathrm{i} \omega_{n}\right)\right] \\
\chi_{(0)+-}^{l l^{\prime}, m m^{\prime}}\left(\mathbf{q}, \mathrm{i} \omega_{m}\right)= & -T \sum_{\omega_{n}, \mathbf{p}, \sigma}\left[G_{m l \uparrow}\left(\mathbf{p}, \mathrm{i} \omega_{n}\right) G_{l^{\prime} m^{\prime} \downarrow}\left(\mathbf{p}+\mathbf{q}, \mathrm{i} \omega_{m}+\mathrm{i} \omega_{n}\right)-\right. \\
& \left.-F_{l m^{\prime} \uparrow}^{\dagger}\left(\mathbf{p},-\mathrm{i} \omega_{n}\right) F_{l^{\prime} m \downarrow}\left(\mathbf{p}+\mathbf{q}, \mathrm{i} \omega_{m}+\mathrm{i} \omega_{n}\right)\right] .
\end{aligned}
$$

Here $G_{m l \uparrow}\left(\mathbf{p}, i \omega_{n}\right)$ and $F_{m l \uparrow}^{\dagger}\left(\mathbf{p}, i \omega_{n}\right)$ are normal and anomalous (Gor'kov) Green's functions.

The physical spin susceptibility $\chi_{+-, z z}\left(\mathbf{q}, \mathrm{i} \omega_{m}\right)=\frac{1}{2} \sum_{l, m} \chi_{+-, z z}^{l l, m m}\left(\mathbf{q}, \mathrm{i} \omega_{m}\right)$ is calculated using Eq. (1) with the interaction matrix $U_{s}$ from [6]. Below we present results that were analytically continued to real frequencies, $\mathrm{i} \omega_{m} \rightarrow \omega+\mathrm{i} \delta$ with $\delta \rightarrow 0+$. We choose the following values for the interaction parameters: spin-orbit coupling constant $\lambda=100 \mathrm{meV}$, intraorbital Hubbard $U=0.9 \mathrm{eV}$, Hund's $J=0.1 \mathrm{eV}$, interorbital $U^{\prime}=U-2 J$, and pair-hopping term $J^{\prime}=J$. Superconducting state is assumed to be either of the $s_{++}$type with the gap function $\Delta_{\mathbf{k}}=\Delta_{0}$ or of the $s_{ \pm}$type with $\Delta_{\mathbf{k}}=\Delta_{0} \cos k_{x} \cos k_{y}$, where $\Delta_{0}=20 \mathrm{meV}$.

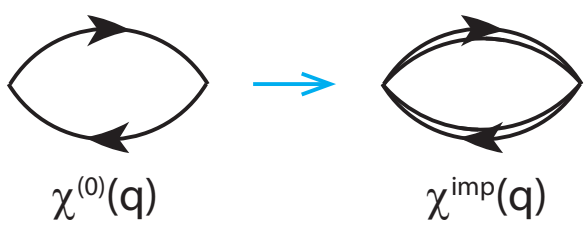

Fig. 3. Replacement of 'bare' Green's functions with the renormalized ones (by the impurity self-energy) in the diagram for the susceptibility $\chi_{(0)}$

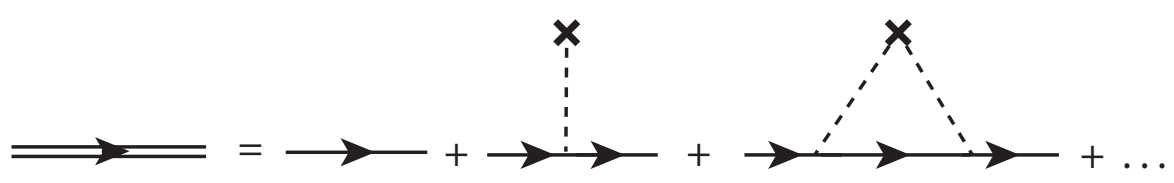

Fig. 4. Impurity self-energy in the static Born approximation

As for the impurity scattering, multiband superconductors demonstrate behavior much more complicated than originally expected from the Abrikosov-Gor'kov theory [22], see Refs. [23-25]. 


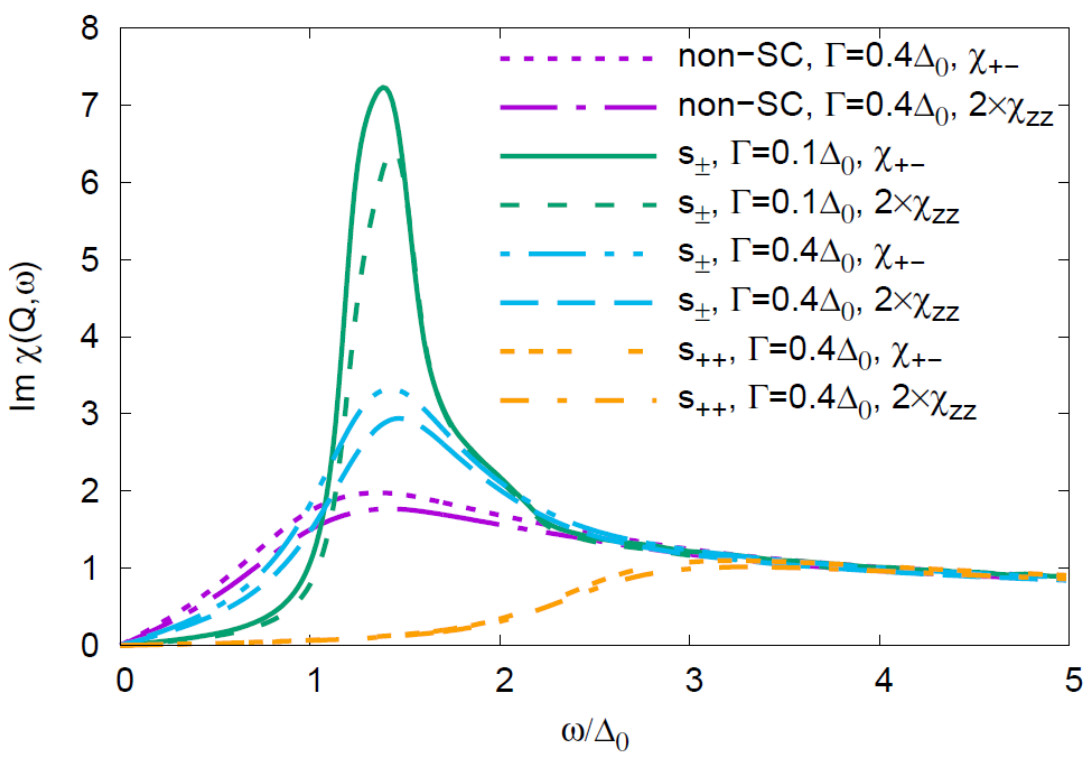

Fig. 5. Calculated $\operatorname{Im} \chi(\mathbf{Q}, \omega)$ with $\mathbf{Q}=(\pi, 0)$ in the 1-Fe $\mathrm{BZ}$ in the normal and superconducting $\left(s_{++}\right.$and $\left.s_{ \pm}\right)$states. In the case of $s_{ \pm}$gap, the peak appears clearly below $\omega=2 \Delta_{0}$

For example, $s_{ \pm} \rightarrow s_{++}$transition takes place for the sizeable intraband attraction in the twoband $s_{ \pm}$model with the nonmagnetic impurities [23] while the magnetic impurities in some cases lead to the $s_{++} \rightarrow s_{ \pm}$transition [25]. Such effects are quite complicated and their treatment require a self-consistent solution of the frequency and gap equations within the $T$-matrix approximation. Here we use a lot simpler approach and stay within the static Born approximation for the quasiparticle self-energy to see the basic effects of nonmagnetic impurities on the spin resonance. The approach is schematically shown in Figs. 3 and 4. The multiple scattering on the same impurity results in the static self-energy $\Sigma(\mathbf{k}) \approx-\frac{\mathrm{i}}{2 \tau_{\mathbf{k}}}$, where $\tau_{\mathbf{k}}$ is the quasiparticle lifetime, see Fig. 4. Calculating the exact momentum dependence of the quasiparticle lifetime is also a separate and complicated task that would require realistic multiorbital models with proper orbital-to-bands contribution [26]. This is again beyond the scope of the present work, so we neglect the momentum dependence of $\tau_{\mathbf{k}}$ and set $\Sigma\left(\mathbf{k}, \mathrm{i} \omega_{n}\right)=-\mathrm{i} \Gamma$, where we treat the impurity scattering rate $\Gamma$ as a parameter.

\section{Results}

Frequency dependence of the +- and $z z$ components of the spin susceptibility for the set of impurity scattering rates $\Gamma$ is shown in Fig. 5. Apparently, $\chi_{+-}>2 \chi_{z z}$ at small frequencies in agreement with results of Ref. [13]. There is a well defined spin resonance peak for the $s_{ \pm}$ superconductor. It is clearly seen below the energy of $2 \Delta_{0}$ in the case of a small $\Gamma$. With increasing $\Gamma$ it becomes broader and almost vanishes once $\Gamma$ becomes comparable to $\Delta_{0}$. For the $s_{++}$state, the difference between $\chi_{+-}$and $2 \chi_{z z}$ is very small. With increasing $\Gamma$, the spin resonance peak broadens and its energy shifts to higher frequencies. The spin response in the $s_{ \pm}$ state is quite different from the spin response in the $s_{++}$state even for a sizeable value of $\Gamma$. Thus 
we demonstrated that the answer to the question whether it is possible to distinguish between $s_{ \pm}$and $s_{++}$states in the presence of nonmagnetic impurities looking at the neutron data is yes, spin responses would be quite different. Another important difference comes from the negligible disparity of $\chi_{+-}$and $2 \chi_{z z}$ components in the $s_{++}$state. This contradicts results of the polarized neutron data [14] thus excluding the $s_{++}$state.

Our findings are in qualitative agreement with the results of Ref. [27] where the band model was simpler but the vertex corrections in the particle-hole bubble due to the impurity scattering were included.

\section{Conclusion}

We studied the simple three-orbital model for FeBS. It's Fermi surface resembles the ones observed in experimental and theoretical studies. We analysed the spin response in the superconducting state in the presence of nonmagnetic disorder and spin-orbit coupling. The disorder was treated within the static Born approximation with impurity scattering rate $\Gamma$ considered as a parameter. For the small $\Gamma$, the spin resonance peak is clearly observed below the energy of $2 \Delta_{0}$. With increasing $\Gamma$, it becomes broader and almost vanishes for a sizeable values of $\Gamma$. The spin resonance peak gains anisotropy in the spin space due to the spin-orbit coupling: for the $s_{ \pm}$superconductor $\chi_{+-}$is larger than $2 \chi_{z z}$. On the other hand, for the $s_{++}$state the disparity between transverse and longitudinal components is negligible. The spin response in the $s_{ \pm}$state is quite different from the spin response of the $s_{++}$superconductor even for large values of $\Gamma$.

\section{References}

[1] M.V.Sadovskii, High-temperature superconductivity in iron-based layered compounds, Physics-Uspekhi, 51(2008), 1201.

[2] D.C.Johnston, The puzzle of high temperature superconductivity in layered iron pnictides and chalcogenides, Advances in Physics, 59(2010), 803.

[3] G.R.Stewart, Superconductivity in iron compounds, Rev. Mod. Phys., 83(2011), 1589.

[4] P.J.Hirschfeld, M.M.Korshunov, I.I.Mazin, Gap symmetry and structure of Fe-based superconductors, Rep. Prog. Phys., 74(2011), 124508.

[5] I.I.Mazin, D.J.Singh, M.D.Johannes, M.-H.Du, Unconventional Superconductivity with a Sign Reversal in the Order Parameter of $\mathrm{LaFeAsO}_{1-x} \mathrm{~F}_{x}$, Phys. Rev. Lett., 101(2008), 057003 .

[6] S.Graser, T.A.Maier, P.J.Hirschfeld, D.J.Scalapino, Near-degeneracy of several pairing channels in multiorbital models for the Fe pnictides, New. J. Phys., 11(2009), 025016.

[7] K.Kuroki, S.Onari, R.Arita, H.Usui, Y.Tanaka, H.Kontani, H.Aoki, Unconventional Pairing Originating from the Disconnected Fermi Surfaces of Superconducting $\mathrm{LaFeAsO}_{1-x} \mathrm{~F}_{x}$, Phys. Rev. Lett., 101(2008), 087004.

[8] S.Maiti, M.M.Korshunov, T.A.Maier, P.J.Hirschfeld, A.V.Chubukov, Evolution of the Superconducting State of Fe-Based Compounds with Doping, Phys. Rev. Lett., 107(2011), 147002. 
[9] M.M.Korshunov, Superconducting state in iron-based materials and spin-fluctuation pairing theory, Physics-Uspekhi, 57(2014), 813.

[10] H.Kontani, S.Onari, Orbital-fluctuation-mediated superconductivity in iron pnictides: analysis of the five-orbital Hubbard-Holstein model, Phys. Rev. Lett., 104(2010), 157001.

[11] M.M.Korshunov, I.Eremin, Theory of magnetic excitations in iron-based layered superconductors, Phys. Rev. B, 78(2008), 140509(R).

[12] T.A.Maier, D.J.Scalapino, Theory of neutron scattering as a probe of the superconducting gap in the iron pnictides, Phys. Rev. B, 78(2008), 020514(R).

[13] M.M.Korshunov, Y.N.Togushova, I.Eremin, P.J.Hirschfeld, Spin-orbit coupling in Fe-based superconductors, J. Supercond. Nov. Magn., 26(2013), 2873.

[14] O.J.Lipscombe, L.W.Harriger, P.G.Freeman, M.Enderle, C.Zhang, M.Wang, T.Egami, J.Hu, T.Xiang, M.R.Norman, P.Dai, Anisotropic neutron spin resonance in superconducting $\mathrm{BaFe}_{1.9} \mathrm{Ni}_{0.1} \mathrm{As}_{2}$, Phys. Rev. B, 82(2010), 064515.

[15] M.M.Korshunov, Y.N.Togushova, I.Eremin, Three-orbital model for Fe-pnictides, J. Supercond. Nov. Magn., 26(2013), 2665.

[16] V.Brouet, M.F.Jensen, P.-H.Lin, A.Taleb-Ibrahimi, P.Le Fevre, F.Bertran, C.-H. Lin, W.Ku, A.Forget, D.Colson, Impact of the two Fe unit cell on the electronic structure measured by ARPES in iron pnictides, Phys. Rev. B, 86(2012), 075123.

[17] A.A.Kordyuk, Iron-based superconductors: Magnetism, superconductivity, and electronic structure, Low Temp. Phys., 38(2012), 888.

[18] I.A.Nekrasov, Z.V.Pchelkina, M.V.Sadovskii, Electronic structure of prototype $\mathrm{AFe}_{2} \mathrm{As}_{2}$ and ReOFeAs high-temperature superconductors: a comparison, JETP Letters, 88(2008), 144.

[19] I.A.Nekrasov, Z.V.Pchelkina, M.V.Sadovskii, Electronic Structure of New LiFeAs High-T Superconductor, JETP Letters, 88(2008), 543.

[20] I.Eremin, D.Manske, K.H.Bennemann, Electronic theory for the normal-state spin dynamics in $\mathrm{Sr}_{2} \mathrm{RuO}_{4}$ : Anisotropy due to spin-orbit coupling, Phys. Rev. B, 65(2002), 220502(R).

[21] K.K.Ng, M.Sigrist, The role of spin-orbit coupling for the superconducting state in $\mathrm{Sr}_{2} \mathrm{RuO}_{4}$, Europhys. Lett., 49(2000), 473.

[22] A.A.Abrikosov, L.P.Gor'kov, Conribution to the theory of superconducting alloys with paramegnetic impurities, Sov. Phys. JETP, 12(1961), 1243.

[23] D.V.Efremov, M.M.Korshunov, O.V.Dolgov, A.A.Golubov, P.J.Hirschfeld, Disorder-induced transition between $s_{ \pm}$and $s_{++}$states in two-band superconductors, Phys. Rev. B, 84(2011), $180512(\mathrm{R})$.

[24] V.G.Stanev, A.E.Koshelev, Anomalous proximity effects at the interface of $s$ - and $s_{ \pm^{-}}$ superconductors, Phys. Rev. B, 86(2012), 174515.

[25] M.M.Korshunov, D.V.Efremov, A.A.Golubov, O.V.Dolgov, Unexpected impact of magnetic disorder on multiband superconductivity, Phys. Rev. B, 90(2014), 134517. 
[26] A.F.Kemper, M.M.Korshunov, T.P.Devereaux, J.N.Fry, H-P.Cheng, P.J.Hirschfeld, Anisotropic quasiparticle lifetimes in Fe-based superconductors, Phys. Rev. B, 83(2011), 184516.

[27] S.Maiti, J.Knolle, I.Eremin, A.V.Chubukov, Effect of nodes, ellipticity, and impurities on the spin resonance in iron-based superconductors, Phys. Rev. B, 84(2011), 144524.

\section{Влияние спин-орбитального взаимодействия и рассеяния на примесях на спин-резонансный пик в трёхорбитальной модели сверхпроводников на основе железа}

Максим М. Коршунов

Юлия Н. Тогушова

Сибирский федеральный университет Свободный, 79, Красноярск, 660041

Россия

Исследуется совместное влияние спин-орбитального взаимодействия и рассеяния на немагнитном беспорлдке на формирование спин-резонансного пика в сверхпроводниках на основе жслеза. Спиновая восприимчивость вычислена в приближении хаотических фаз. Спин-резонансный пик становится шире с увеличением беспорядка, и его частота также смещается. В то же время спиновый отклик в состоянии $s_{ \pm}$отличается от такового в состоянии $s_{++}$.

Ключевые слова: сверхпроводники на основе железа, спин-орбитальное взаимодействие, рассеяние на примесях, спин-резонансный пик. 